\title{
A search for magnetic fields on central stars in planetary nebulae
}

\author{
F. Leone ${ }^{1}$, R. L. M. Corradi ${ }^{2,3}$, M. J. Martínez González ${ }^{2,3}$, A. Asensio Ramos ${ }^{2,3}$, and R. Manso Sainz $z^{2,3}$ \\ ${ }^{1}$ Università di Catania, Dipartimento di Fisica e Astronomia, Sezione Astrofisica, via S. Sofia 78, 95123 Catania, Italy \\ e-mail: fleone@oact.inaf.it \\ 2 Instituto de Astrofísica de Canarias, 38205 La Laguna, Tenerife, Spain \\ ${ }^{3}$ Departamento de Astrofísica, Universidad de La Laguna, 38205 La Laguna, Tenerife, Spain
}

Received 26 September 2013 / Accepted 20 January 2014

\begin{abstract}
Context. One of the possible mechanisms responsible for the panoply of shapes in planetary nebulae is the presence of magnetic fields that drive the ejection of ionized material during the proto-planetary nebula phase.

Aims. Therefore, detecting magnetic fields in such objects is of key importance for understanding their dynamics. Still, magnetic fields have not been detected using polarimetry in the central stars of planetary nebulae.

Methods. Circularly polarized light spectra have been obtained with the Focal Reducer and Low Dispersion Spectrograph at the Very Large Telescope of the European Southern Observatory and the Intermediate dispersion Spectrograph and Imaging System at the William Herschel Telescope. Twentythree planetary nebulae that span very different morphology and evolutionary stages have been selected. Most of central stars have been observed at different rotation phases to point out evidence of magnetic variability.

Results. In this paper, we present the result of two observational campaigns aimed to detect and measure the magnetic field in the central stars of planetary nebulae on the basis of low resolution spectropolarimetry. In the limit of the adopted method, we can state that large scale fields of $\mathrm{kG}$ order are not hosted on the central star of planetary nebulae.
\end{abstract}

Key words. techniques: polarimetric - stars: magnetic field - planetary nebulae: general

\section{Introduction}

In the past twenty years, extensive ground-based and HST imaging has revealed the extraordinary shapes of planetary nebulae (PNe). The original paradigm of PNe as spherical shells that expand uniformly around a stellar remnant, a hot white dwarf, is clearly far from describing reality. We now know that different kinds of geometries, which range from spherical or ellipsoidal to highly collimated ones, characterize the overall shape of PNe (e.g. Corradi \& Schwarz 1995). A rich realm of additional structures, such as symmetrical (or not) pairs of knots, jets, ansae, etc., is found on smaller scales embedded in the main bodies or located externally from them (Gonçalves et al. 2001).

These articulated morphologies clearly indicate that mass loss at the very end of the asymptotic giant branch (AGB) is complex and still far to be fully understood. Presently, a strong dynamical interaction between the massive/slow AGB wind (produced by surface levitation of gas due to convection, stellar pulsations, and radiation pressure on dust) and the fast/tenuous post-AGB wind (driven by blanketed UV absorption lines of ions) is thought to play a basic role in governing the formation and evolution of PNe, but it does not explain the deviation from the spherical geometry. To account for it, a number of explanations have been proposed (see, e.g. Balick \& Frank 2002) with the most popular ones being interactions in mass-exchanging binary systems or the emergence of surface magnetic fields. Such models have one preferred symmetry axis and, hence, might account for simple bipolar flows, but to reproduce all multi-polar structures (like multi-lobal nebulae or multiple blobs and jets) the theoretical scenario had to be enriched with other ingredients able to break the axial symmetry (like precession of accretion disk winds or off-axis magnetic fields).
The binary scenario has been explored both theoretically (e.g., Soker \& Rappaport 2001) and observationally (e.g., Miszalski 2012; Boffin et al. 2012), and while it can explain a number of structures observed in the nebulae, it is unlike gravitational interactions are sufficient to explain all the variety of outflows found in the same nebula (see e.g., the case of Mz3 in Santander et al. 2004, where four distinct flows with increasing collimation degree have been identified).

Magnetic fields can provide the additional mechanisms needed to explain the observed structures. With this aim, a variety of magneto-hydrodynamical (MHD) simulations of the nebular shaping have appeared in literature (e.g. Garcia-Segura et al. 1999; Matt et al. 2004; Frank \& Blackman 2004). The magnetic field may be either a fossil remnant from the progenitor on the main sequence (e.g., Ap stars) or can be generated by a dynamo at the interface between a rapidly rotating stellar core and a more slowly rotating envelope. Blackman et al. (2001) argue that some remnant fields anchored in the core survive even without a convection zone, although the convective envelope may not be removed completely. Thomas et al. (1995) have shown that white dwarfs, which do have thin surface convection zones, can support a near-surface dynamo. Since the field strength in their model is higher at higher luminosities, this would particularly be true for central stars of PNe. That some central stars should contain significant magnetic fields is also indicated by the presence of magnetic fields, between $10^{3}$ and $10^{9}$ Gauss, in some $10-30 \%$ of all white dwarfs.

In spite of these facts and of MHD simulations, that are quite successful in reproducing several of the observed nebular structures; to date, very little observational evidence has been obtained of the existence of such magnetic fields. Still, magnetic fields have not been detected in the central stars of 
planetary nebulae. First positive detections at a kiloGauss level were claimed by Jordan et al. (2005) in two PNe. However, Leone et al. (2011), Bagnulo et al. (2012), and Jordan et al. (2012) could not confirm these results.

Jordan et al. (2012) have measured the magnetic field in the central stars of eleven planetary nebulae, concluding that there is still no evidence for the existence of magnetic fields in PN central stars to date. In this paper, we continue our search for magnetic fields in PN central stars via spectropolarimetry, as done in Leone et al. (2011). The survey is not focussed on specific morphological classes but should be considered as a panoramic view with the main goal of highlighting the overall properties of magnetic fields (if any) in the central stars of PNe in general. Detecting magnetic fields in one or the other of the observed morphologies would demonstrate that magnetic fields are indeed at work and would start revealing which kind of shaping processes they are relevant for. With the aim to enlarge our sample, and for an omogeneousy reduction and analysis, raw spectropolarimetric data of further seven central stars observed with the FOcal Reducer and low dispersion Spectrograph (FORS) have been obtained from the archive of the European Southern Observatory.

\section{Observations, data reduction, and magnetic field measurements}

Measuring stellar magnetic fields is one of the most demanding techniques because of the need to reach very high signalto-noise ratios. In the weak-field approximation for stellar atmospheres (Landstreet 1982; Mathys 1989), the disk-integrated Stokes- $V$ parameter (the difference between the opposite circular polarized intensities) across spectral line profiles is proportional to the longitudinal component of the magnetic field integrated over the stellar disk, or the so-called effective magnetic field. High-resolution circular spectropolarimetry gives the possibility to distinguish photospheric regions with positive and negative magnetic fields (Leone \& Catanzaro 2004; $R=115000$ ). Circular spectropolarimetry is also useful at moderate resolution (Leone \& Catanzaro 2001; $R=15000$ ) for detecting magnetic fields, but it is still prohibitive for faint stars. As for white dwarfs, Angel \& Landstreet (1970) introduced a method based on narrowband ( $\sim 30 \AA$ ) circular photopolarimetry on the wings of the $\mathrm{H}_{\gamma}$ Balmer line. Bagnulo et al. (2002) have shown how to co-add the Stokes- $V$ signal from spectral lines, as observed at low resolution, and measure the effective field of spectra lines on very faint stars.

With the aim to measure the effective magnetic field of the central stars of planetary nebulae, we have adopted the procedures and methods presented in Leone et al. (2011). Because of the necessary huge signal-to-noise ratio $(S / N \sim 5000$ was achived for an upper limit of $300 \mathrm{G}$ in the case of PNe NGC1360), we have selected PNe among the brightest ones that cover a range of morphologies as large as possible. Another important aspect to detect magnetic fields with the adopted technique is to select targets for which the nebular Balmer emission is negligible long the line of sight of the central star.

Spectropolarimetric data have been collected 1) at the William Herschel Telescope (WHT) at the Observatorio del Roque de los Muchachos at La Palma, Spain, using the ISIS spectrograph and 2) at the Unit 1 of the Very Large Telescope at ESO, Chile, using FORS2. With ISIS, data were obtained in the 3785-4480 ̊ range at resolution $R=5000$ with the procedures described in Leone (2007). As to FORS2, data were obtained in the 3800-5200 $\AA$ range at a resolution $R=2700$ with the procedures described in Leone et al. (2011). Information about the basic properties of each target PN, a log-book of the observations, and obtained results are listed in Table 1.

To increase the signal-to-noise level of the polarized spectra, we averaged the observations of the same object that were not separated more than half the period of rotation of the object. Each averaged observation has been labeled with a number from 1 to 10. The reduction and the demodulation procedure are the same for all objects (see Leone et al. 2011). Figure 1 shows the Stokes $I$ spectra, as observed with FORS2 and WHT. The last column of Table 1 shows the results of our spectropolarimetric analysis. No magnetic field has been detected on the central stars of the selected planetary nebulae. It is worthy to note that no analysis could be done either for nine targets because the nebular contamination was too high: NGC 246, PN G24337.1, NGC 3242, He2-138, Hen 2-194, ESO588-14, NGC 6629, IC 4776, and NGC 7009, as seen in the lower panels of Fig. 1.

\section{Discussion}

Although Despite we have no positive detections of a magnetic field in any of the observed PNe, this is significant result, since targets span a large range of nebular and stellar parameters (Table 1). In particular, a variety of morphological structures seen at different inclination angles are included from the marked bipolar shape of HD 44179 (the Red Rectangle), the mild bipolar morphology of NGC 4361, the ring/disc like inner nebula of NGC 7293 with outer multipolar lobes, and the elongated geometry of NGC 1360, for example, to the almost perfectly spherical shape of nebulae, like Tc 1 . In addition, highly collimated structures, which might be related to magnetic shaping, are also present, such as the highly inclined jets of NGC 1360, the poleon ones of NGC 2392, or the symmetric pair of low-ionization knots of NGC 6826. The only morphological class, which is not well represented (except for the case of the pre-PN HD 44179) is that of classical "butterfly" nebulae with a narrow waist from which high velocity bipolar lobes depart (see e.g., Corradi \& Schwarz 1995). While their extreme collimation put them at among the most promising targets for magnetic fields (Sabin et al. 2007), they are beyond the reach of the method adopted in this paper. One reason is that that their central stars are often intrisically faint, because they are relatively massive and therefore have a fast post-AGB evolution towards low luminosities (Corradi \& Schwarz 1995). In addition, they have dense equatorial torii of gas/dust, whose emission/absorption often prevents the observations of the central stars.

Some binary central stars, which can naturally provide an additional mechanism to produce magnetic fields (such as angular momentum transfer to one of the two stellar components) are also included. The object NGC 6026 is a close binary with a period of 0.53 day (Hillwig 2010), composed of two degenerate compact stars, which experienced a common-envelope phase before ejecting the observed nebula and jets. The period of NGC 1514 is not known, but the presence of an A-type star at its centre, which is too cool to produce the ionization of the nebula, indicates that it is also a binary system with a much likely longer period. This occurs because no large radial velocity shifts has been detected so far (De Marco et al. 2004). As the A-type companion star dominates the spectrum in the visual range, our study effectively looks for a magnetic field in the companion rather than in the star that ejected the PN. This is still interesting, as the possibility of an induced magnetic field in the companion stars, or even of a common magnetosphere involving the 
Table 1. Main properties of the target PNe. The $B_{\text {mag. }}$ of the central star is reported.

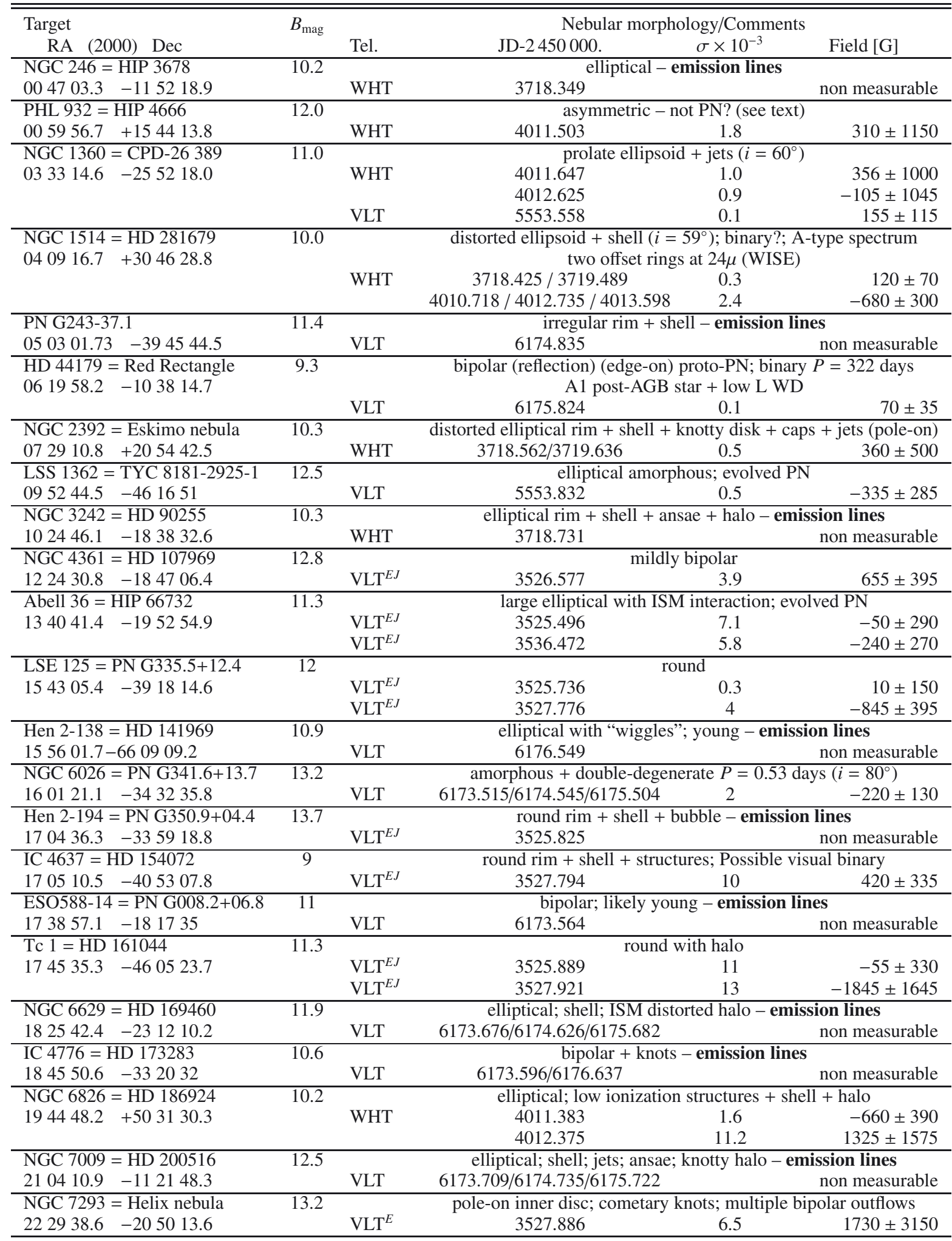

Notes. The present method, the magnetic field could not be measured in stars with a nebular emission overimposed to Balmer lines. ${ }^{(E)}$ ESO Archive data. ${ }^{(J)}$ Also Jordan et al. (2012).

two stars (e.g., $\beta$ Lyrae, Leone et al. 2003), exists. Note also the longer period of HD 44179 (322 days) and the possible very long period binary nature of IC 4637 (De Marco et al. 2004).
Target nebulae also peaked up at different evolutionary stages from the pre-PN nature of HD 44179 to the very evolved nebulae of LSS 1362 and Abell 36. In this respect, it should be 

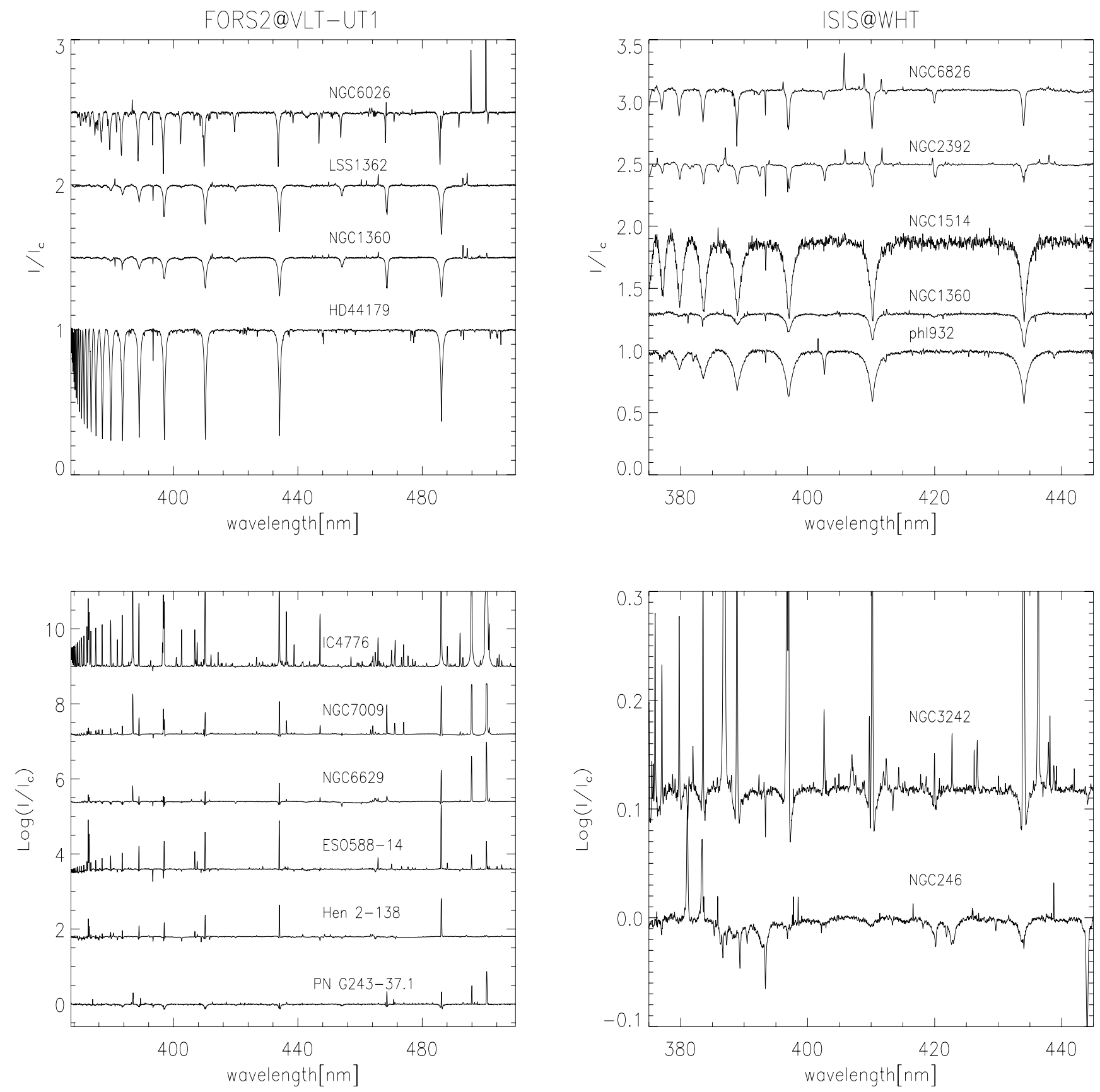

Fig. 1. Observed central stars of planetary nebulae listed in Table 1. Left panels show the stars observed with FORS2 at UT2. Right panels show the stars observed with ISIS at WHT. Low panels show the logarithmic relative intensity for those stars dominated by emission lines. An ad hoc shift has been assumed to avoid the overlapping of spectra.

noted that the nebula observed around PHL 932 might not be a $\mathrm{PN}$ but is just ambient medium ionized by a hot sdB star, or a Strömgren sphere (Frew et al. 2010).

Although our search for magnetic fields has addressed a variety of morphological structures and central-star parameters/duplicity, which are often been related to magnetic fields, we still have no evidence of magnetic fields of the order of a $\mathrm{kG}$ or less in PN central stars. If the central stars of the PNe reported here are all characterised by the same dipolar strength with a magnetic axis randomly oriented with respect to the line of sight, then there is the $95 \%$ probability that their dipolar strength is $<800 \mathrm{G}$ (see Kolenbergen \& Bagnulo 2009). Using Jordan et al. (2012) data, this upper limit is $1100 \mathrm{G}$. A forthcoming paper where a rigorous statistical analysis of $\mathrm{PNe}$ observed so far from different groups will be presented (Asensio Ramos et al. 2014). Future efforts will be directed to detect these fields in the nebular gas and dust, which will allow us to explore different types of targets and nebular parameters.

Acknowledgements. A.A.R., M.J.M.G. and R.M.S. acknowledge financial support by the Spanish Ministry of Economy and Competitiveness (MINECO) through project AYA2010-18029, and RLMC through proyect AYA2012-35330. A.A.R. and R.M.S. acknowledge financial support by the MINECO through the project Consolider-Ingenio 2010 CSD2009-00038. This paper is based on observations made with the WHT operated on the island of La Palma by the ING in the Spanish Observatorio del Roque de los Muchachos of the Instituto de Astrofísica de Canarias. It is also based on observations made with ESO Telescopes at the La Silla Paranal Observatory under programme ID 089.D-0429(A). We thanks the referee, Dr. Stefano Bagnulo, for suggestions and comments. 
F. Leone et al.: A search for magnetic fields on central stars in planetary nebulae

\section{References}

Angel, J. R. P., \& Landstreet, J. D. 1970, ApJ, 160, L147

Bagnulo, S., Szeifert, T., Wade, G. A., Landstreet, J. D., \& Mathys, G. 2002, A\&A, 389, 191

Bagnulo, S., Landstreet, J. D., Fossati, L., \& Kochukhov, O. 2012, A\&A, 538, 129

Balick, B., \& Frank, A. 2002, ARA\&A, 40, 439

Blackman, E. G., Frank, A., Markiel, J. A., Thomas, J. H., \& Van Horn, H. M. 2001, Nature, 409, 485

Boffin, H., Miszalski, B., Rauch, T., et al. 2012, Science, 337, 773

Corradi, R. L. M. 2006, IAU Symp. 234 (Cambridge University Press), 277

Corradi, R. L. M., \& Schwarz, H. E. 1995, A\&A, 293, 871

Corradi, R. L. M., Livio, M., Balick, B., Munari, U., \& Schwarz, H. E. 2001, ApJ, 553, 211

De Marco, O., Bond, H., Harmer, D., \& Fleming, A. J. 2004, ApJ, 602, L93

Frank, A., \& Blackman, E. 2004, ApJ, 614, 737

Frew, D. J., Madsen, G. J., O’Toole, S. J., \& Parker, Q. 2010, PASA, 27, 203

Garcia-Segura, G., Langer, N., Rozyczka, M., \& Franco, J. 1999, ApJ, 517, 767

Gonçalves, D. R., Corradi, R. L. M., \& Mampaso, A. 2001, ApJ, 547, 302
Hillwig, T. C., Bond, H. E., Afsar, M., \& De Marco, O. 2010, AJ, 140, 319 Jordan, S., Werner, K., \& O'Toole, S. J. 2005, A\&A, 432, 273

Jordan, S., Bagnulo, S., Werner, K., \& O’Toole, S. J. 2012, A\&A, 542, A64

Kolenbergen, K., \& Bagnulo, S. 2009, A\&A, 498, 543

Landstreet, J. 1982, ApJ, 258, 639

Leone, F. 2007, MNRAS, 382, 1690

Leone, F., \& Catanzaro, G. 2001, A\&A, 365, 118

Leone, F., \& Catanzaro, G. 2004, A\&A, 425, 171

Leone, F., Plachinda, S. I., Umana, G., Trigilio, C., \& Skulsky, M. 2003, A\&A, 405, 223

Leone, F., Martínez González, M. J., Corradi, R. L. M., Privitera, G., \& Manso Sainz, R. 2011, ApJ, 731, 33

Mathys, G. 1989, Fund. Cosm. Phys., 13, 14

Matt, S., Frank, A., \& Blackman, E. G. 2004, ASP Conf. Proc., 313, 449

Miszalski, B. 2012, IAU Symp., 283, 107

Sabin, L., Zijlstra, A. A., \& Greaves, J. S. 2007, MNRAS, 376, 378

Santander-García, M., Balick, B., Corradi, R. L. M., \& Mampaso, A. 2004, A\&A, 426, 185

Soker, N., \& Rappaport, S. 2001, ApJ, 557, 256

Thomas, J. H., Markiel, A., \& Van Horn, H. M. 1995, ApJ, 453, 403 